JAIST Repository

https://dspace.jaist.ac.jp/

\begin{tabular}{|c|c|}
\hline Title & $\begin{array}{l}\text { Pract i cal Envi ronment for Real i zi ng Augment ed } \\
\text { Cl assroom wi th Wr rel ess Di gi tal Pens }\end{array}$ \\
\hline Author(s) & $\begin{array}{l}\text { M ur a, Not oki ; Kuni fuj i , Susumi; Sakanmt o, } \\
\text { Yasuyuki }\end{array}$ \\
\hline Citation & $\begin{array}{l}\text { Lect ure Notes in Computer Sci ence, 4694/2007: } \\
777-785\end{array}$ \\
\hline Issue Date & 2007 \\
\hline Type & Journal Article \\
\hline Text version & aut hor \\
\hline URL & ht t p: //hdl . handl e. net /10119/4472 \\
\hline Rights & 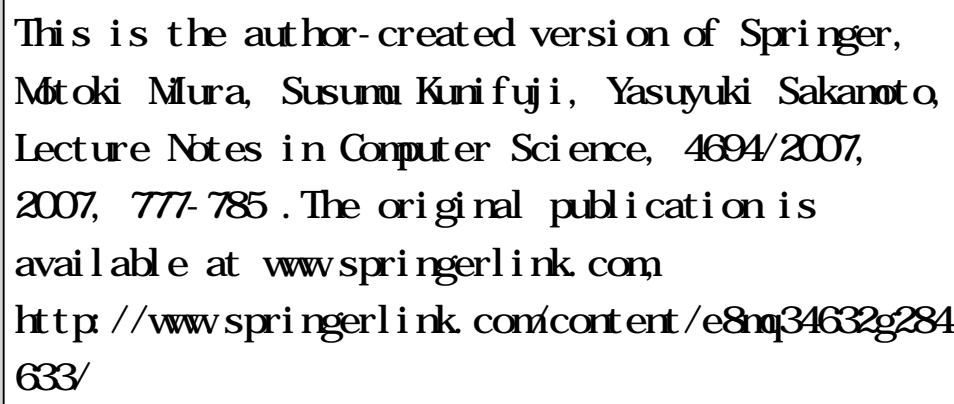 \\
\hline Description & \\
\hline
\end{tabular}

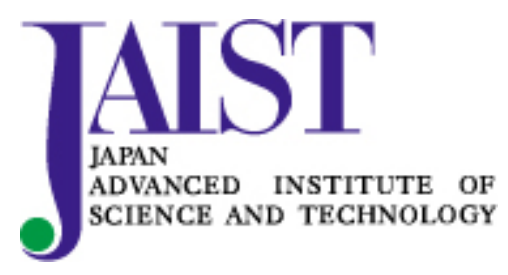




\title{
Practical Environment for Realizing Augmented Classroom with Wireless Digital Pens
}

\author{
Motoki Miura $^{\dagger}$, Susumu Kunifuji ${ }^{\dagger}$, and Yasuyuki Sakamoto ${ }^{\dagger \dagger}$ \\ $\dagger$ School of Knowledge Science \\ Japan Advanced Institute of Science and Technology \\ \{miuramo,kuni\}@jaist.ac.jp \\ http://css.jaist.ac.jp/ miuramo/ \\ $\dagger \dagger$ Senior High School at Sakado, University of Tsukuba
}

\begin{abstract}
We have developed AirTransNote, a student notes sharing system to facilitate collaborative and interactive learning in a regular lecture at conventional classrooms. Our former student system employed ultrasonic digital pens to capture student notes on a usual paper sheet. Although the paper-based approach is intuitive for learners, the ultrasonic pen with a PDA created some difficulties when used by senior high school students. In order to eliminate those difficulties, we have introduced anoto-based pens and a data gathering system called "digital pen gateway." Owing to the improved student system, students could easily submit their answers with handling of multiple paper sheets. The teacher could refer the answers to choose students who need assistance during the lecture. Questionnaire results showed that the simpler student interface is quite acceptable, and the system can realize our augmented classroom concept in a practical way.
\end{abstract}

\section{Introduction}

Mobile computing and wireless networking technologies have been applied to facilitate computer-supported collaborative learning (CSCL) not only in university and collage courses[1-3] but also in younger students' learning activities outdoor[4] or in museum[5]. Most of the CSCL projects employ mobile/handheld devices such as Tablet PCs, PDAs, and cellular phones as a main interface for students to collect students' activities. However the interface and media should be carefully designed to assist learning especially for lower-performing students[6].

We have previously developed "AirTransNote (ATN)," a student note sharing system to facilitate collaborative and interactive learning in a regular lecture at conventional classrooms[7]. In that work, we have chosen a digital pen, which can capture student notes written on regular paper, and PDA to transmit the notes immediately to a teacher's PC. The teacher could browse the notes, and show them to the students. Also our ATN could provide feedback to students based on the notes. When using ATN, no computer literacy was required since the writing task was quite popular. 
Although the natural and intuitive design of ATN with digital pen was effective for student learning, some difficulties of former student system had been exposed in our feasibility studies. In order to eliminate those difficulties, we have improved the student system of ATN and the system's architecture. In this paper, we describe that improvement and discuss the new system's feasibility based on the results of experimental lecture.

\section{Difficulties of Former Student System}

Before explaining the difficulties, we have to describe our former student system in detail. The former student system consisted of an ultrasonic digital pen and a WiFi-enabled PDA (see Fig. 1). The ultrasonic wave diffused from the pen tip was detected by a clip sensor attached above the paper. The sensor estimated the position of the pen tip based on the ultrasonic wave. The position data was transmitted to the PDA via IrDA, and then re-transmitted to the teacher's PC via WiFi connection (see Fig. 2).

The ultrasonic digital pen has the advantage of handling any type of paper sheet. However, it presents problems in accuracy and stability due to its intrinsic mechanism. Firstly, in order to keep consistency between paper and digital notes, the student needs to calibrate it before writing. Even having designed a simple calibration process ${ }^{1}$, we found that some high school students had difficulties in it. Also, once the calibration has been understood, some students still put their hands in front of the clip sensor, which prevented an accurate position sensing. We have provided a confirmation monitor which displays their notes on the personal PDA screen, and that reduced the calibration errors to some extent. But we could not eliminate errors completely, and that made precision rates of region-based handwritten recognition lower.

To make matters worse, the student system required the calibration process not only at the initial setup but also after exchanging the sheet for students. In addition to the calibration, students were asked to tell the system which paper sheet was currently being set, by pressing PDA buttons. This step was easily forgotten. In general, using multiple sheets in one lecture is quite effective because the teacher can provide enough margins on the sheets, and the margins can be utilized to rewrite correct answers instead of erasing ink ${ }^{2}$. But the consistency between paper sheets and digitized notes was often violated by those missing operations.

Secondly, the separate subcomponents of the digital pen caused trouble both in setup and working phase. The students had to setup the system as shown in Fig. 1 by connecting and placing the sensor device and the PDA. Even though we provided a visual reference sheet to explain the setup process, the complicated

\footnotetext{
${ }^{1}$ The system asks the user to tap three points on the paper in sequence (left side, bottom-left, and bottom right) to determine the relative position of the paper and clip sensor.

${ }^{2}$ The digital pen adopts a ball-point pen, so notes cannot be erased or modified.
} 
configuration was troublesome for some students. In addition, students and system maintainers had to mind the battery status for all separated subcomponents (a pen, a transmitter and a PDA) during the lecture.

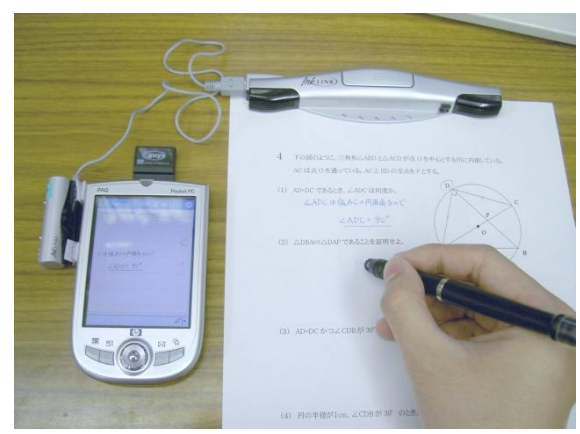

Fig. 1. Former Student System (Ultrasonic Digital Pen and PDA)

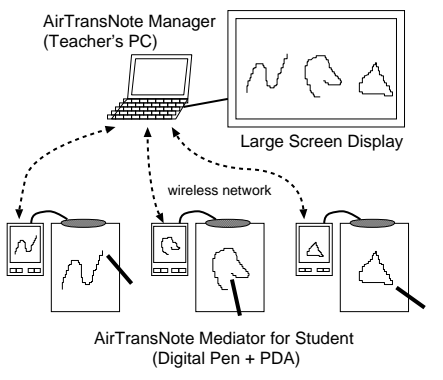

Fig. 2. Former System Configuration

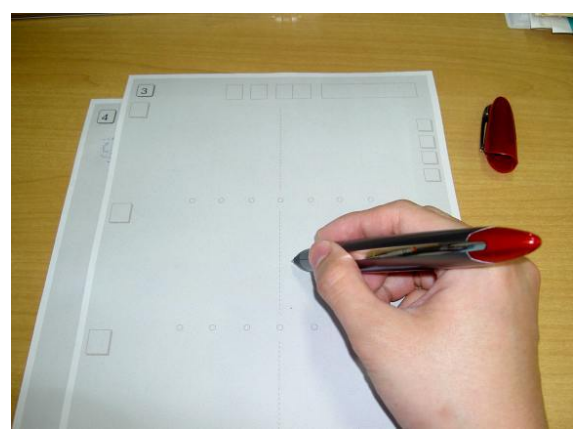

Fig. 3. Current Student System (Anoto Pen and Papers)

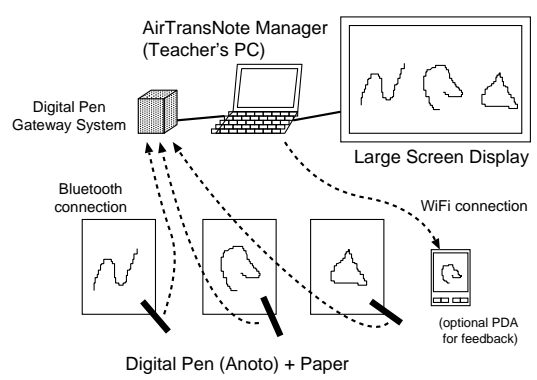

Fig. 4. Current System Configuration

\section{New Architecture of AirTransNote}

Some of the difficulties mentioned could be overcome if students got accustomed to the student system including the characteristics of the ultrasonic pen device. However, training is an additional burden on students, and it is troublesome to have them make time for acquiring skills to handle the devices.

In order to minimize the hurdles, we have introduced a new ATN architecture by adopting anoto-based digital pens and a wireless data gathering system named "digital pen gateway." An anoto-based digital pen (anoto pen) has the capability to store and send handwritten notes made on a specific dotted paper sheet. A camera embedded in the pen tip continuously captures images of the small dots. The captured image is used to calculate the position of the pen tip. With the anoto pen, the student is free of the setup and calibration process. Also, the 
Table 1. Comparison of student note capturing systems

\begin{tabular}{|c|c|c|}
\hline item & Ultrasonic pen and PDA & Anoto pen \\
\hline \hline Setup & hold paper by clip sensor and connect & none \\
\hline Calibration & tap three points on the paper & none \\
\hline Error & approx. 0-20mm (caused by obstacle) & none in principle \\
\hline Send Cue & automatic (when the pen tip left) & manual (tap on "SEND" box) \\
\hline Wireless & IEEE802.11b (embedded in PDA) & Bluetooth (embedded in Pen) \\
\hline Available sheet & any & specific dotted paper sheet \\
\hline Multiple sheets & manual set on PDA & automatically detect \\
\hline
\end{tabular}

teacher can collect accurate and stable student notes. The anoto pen can detect different paper sheets if a distinct dot pattern is printed and registered. Thus the student can handle multiple sheets without any additional operations or concern. A typical scene of using the anoto pen is shown in Fig. 3. Comparing it to Fig. 1, the number of necessary subcomponents is reduced from four to one. Consequently, the setup procedures, such as connecting physical cables, are eliminated. Tab. 1 explains the differences of the student systems.

The anoto pen data is usually transmitted to a device such as a PC or a PDA through a USB cradle or a point-to-point Bluetooth connection. It is possible to use a student PDA to mediate data transmission, but the number of intermediate hardware should be minimized to reduce trouble. Thus we chose a digital pen gateway system solution developed by NTT Comware Corporation ${ }^{3}$. The gateway system is designed to receive pen data through Bluetooth wireless connections and can handle about forty pens simultaneously. The new system's configuration is shown in Fig. 4. Student PDAs can be added to provide confirmation and feedback, if necessary; teachers can decide if such feature is required or not.

The former system could transmit notes immediately with no additional operations. However, the current anoto pen only transmits them when the student explicitly taps a specific region of the paper registered as "send." This limitation may cause some delay on notes update, which is unfavorable to our initial objective. But we regard the merits of accuracy and stability of notes and easy setup as more important than immediate and automatic update. If the students can be naturally aware of the sending status, they will not be bothered because the sending operation is simple.

\section{Experimental Lecture}

We conducted experimental lectures at a senior high school to check the feasibility of the new architecture in a real classroom setting. For that matter, we needed to compare the new system with the former one. Therefore we chose a mathematics lecture in a 40-members class that had experienced the previous

\footnotetext{
${ }^{3}$ http://www.nttcom.co.jp/english/news/en07021301.html
} 
ultrasonic pen. All students had experienced the earlier system, and half of the students had used the system in a mathematical lecture.

We presented forty anoto pens, the digital pen gateway system, a teacher's $\mathrm{PC}$, and a projector with screen to the classroom. At the beginning of the lecture, we distributed one anoto pen and three worksheets to each student. We asked them to pick the pen with their student ID in it, so that notes could be properly identified. We also had the students' seat layout configured prior to the lecture, so that the system displayed their notes according to it.

The students first worked on problems in the worksheets with digital pens, and then the teacher explained selected questions by zooming in on students' answers. Each worksheet included two or three math problems and corresponding answering boxes. Students were asked to solve the problems with the pen, and to write the solutions in the answering boxes. The solutions were recognized by ATN Manager. If the answer was correct, the corresponding area of the student note was painted green, otherwise red (see Fig. 5). Thus both students and teacher could recognize whether answers were correct or not by just seeing the color. Students can easily proceed to the next problem if they get correct feedback. Also the teacher can immediately identify students who need his/her assistance. We performed the forty-five minutes lecture twice in the same class on two different days (Tuesday and Wednesday). Also we conducted a questionnaire survey at the following math class on the Friday of the same week.

\subsection{Result}

The students could easily understand how to use the anoto pen, and used their notes for checking answers. Fig. 5 shows scene of our experimental lecture. The students and the teacher could refer the progress by the students' notes displayed on the shared screen. Some of the handwritten numbers were not recognized properly due to irregular writing sequence. The recognition rate was improved after instructing the regular sequence. They could know whether the note was properly sent or not through pen vibration, as well as the student ID color changing to yellow.

Fig. 6 shows the results of the questionnaire survey compared to the former system. We compared the result distribution with previous data, answered by students who used ultrasonic and PDA system in regular math class (introducing extended definitions of trigonometric functions where exceed 90 degrees[8]). According to the chi-square test, the rating distributions differed significantly in two of the items: "Q2: The pen was useful for lecture." $\left(\chi^{2}(3)=8.10, \mathrm{p}=.044\right)$, "Q5: I got nervous by teacher's check." $\left(\chi^{2}(3)=8.85, \mathrm{p}=.031\right)$. Fig. 7 shows the comparison of attitudes towards each of the two systems. According to the chisquare test, the rating distributions of three items differed significantly: "Q9: Did you enjoy the lecture?" $\left(\chi^{2}(2)=10.1, \mathrm{p}<.010\right)$, "Q10: Did you understand the contents?" $\left(\chi^{2}(3)=10.1, \mathrm{p}=.018\right)$, "Q13: Did you actively participate to the class?" $\left(\chi^{2}(3)=7.41, \mathrm{p}=.025\right)$. Considering the Q2 result, students who felt the pen was useful for the lecture increased because of the new system's simplicity. Also, most of the students enjoyed the lecture with the system (Q9), and 


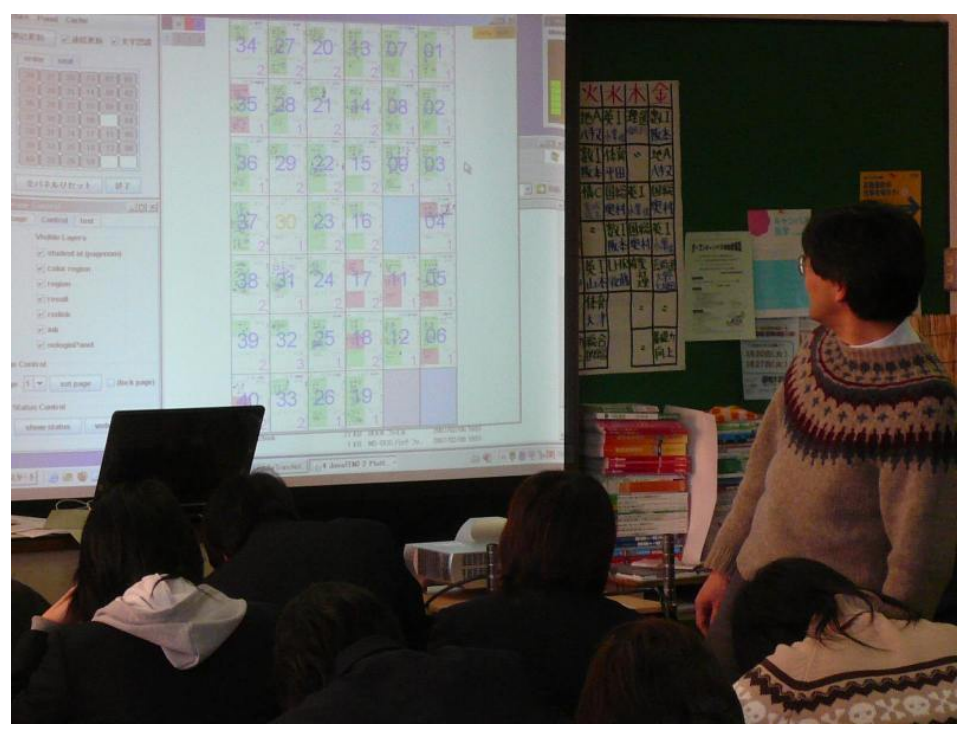

Fig. 5. Scene of the experimental lecture

the note sharing function improved their in-class participation (Q13). Our questionnaire survey did not include items that ask the difference of the pen. But students apparently scored the difference on the general items on system. Many students said that he/she had a good time with fun. These results support the usability and practical aspects of the system. But some students complained of the precision of handwritten recognition. We chose an online recognition module, but students tended to overwrite numbers to make the character solid and bold. This problem would be relieved by adopting an offline recognition module.

Regarding Q5, uneasy feeling in sharing note may be relieved by notes stability or other factors. Since the results of Q5 and Q10 may depend on the difficulty of lecture contents, further investigation is required to clarify those factors.

\section{Related Works}

There is varied research on enriching classroom facilities by embedding devices. Cooperstock developed a computer-augmented classroom with automated controls and lecture capturing capabilities by coordinating room-light, projectors, and white boards[9]. The Smart Remote Classroom [10] system aims to support distributed learning in terms of network technology and ubiquitous environment. EUME [11] system is a task-based PDA interface for teachers to manage computer-augmented classroom which includes a projector and an electric white board. We intended to augment real-time in-class participation by bringing mobile appliances, without any changes to the classroom interior.

Liu et al. argued about learning environment with handheld devices and large shared screen for group communication[12]. They focused on the effects of shared 

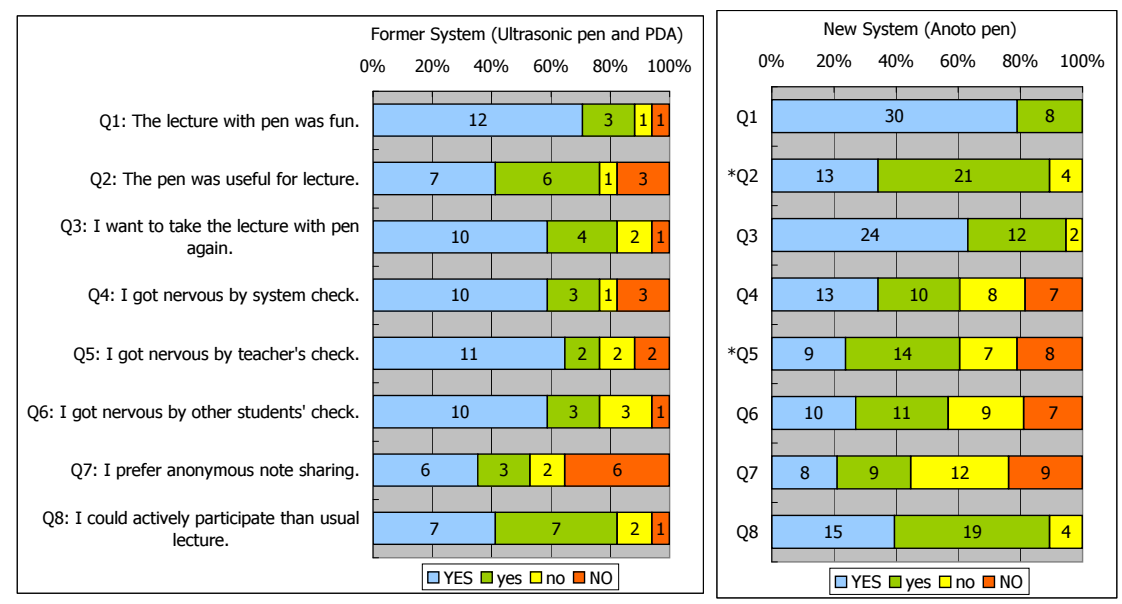

Fig. 6. Questionnaire about the system (* denotes $5 \%$ significant)
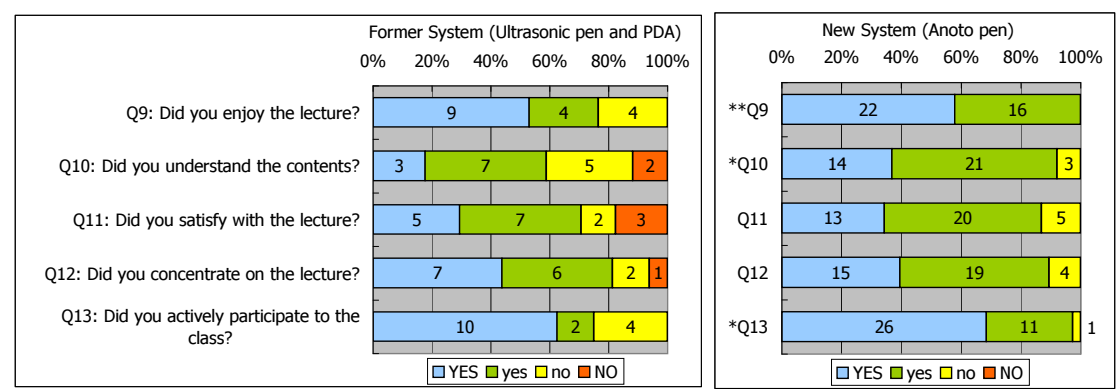

Fig. 7. Questionnaire about attitude for lecture $\left(*\right.$ denotes $5 \%$ significant, ${ }^{* *}$ denotes $1 \%$ significant)

screen on communication patterns in a small group. Our system also utilize a large shared screen for understanding students' progress, but we aim to facilitate efficient and interactive lecture with dynamic capturing of notes.

\section{Conclusion and Future Works}

In this paper, we described the improvement in an earlier ATN system based on a change in the student devices. The simplicity in both the architecture and the calibration-free digital pen contributes to a higher usability of the lecture with note sharing in a regular classroom. Also, we confirmed its feasibility by conducting experimental lectures. We believe our system became quite practical to enhance lectures in conventional classrooms.

In this experiment, we adopted anoto pens to capture students' notes, but we do not stick to that particular capturing device. We believe that any pen 
devices that captures accurate and stable notes, and supports multiple paper sheets without much concern, can be successfully used.

We will investigate the learning effect of the system through long term experiments. We will also continue to improve the system to bring better learning experiences for both teachers and students by developing an analysis technique of online student notes.

\section{Acknowledgment}

Part of this work was supported by a grant-in-aid for Scientific Research (15020216, 17011028).

\section{References}

1. Anderson, R.J., Hoyer, C., Wolfman, S.A., Anderson, R.: A Study of Digital Ink in Lecture Presentation. In: Proceedings of CHI 2004. (2004) 567-574

2. Kam, M., Wang, J., Iles, A., Tse, E., Chiu, J., Glaser, D., Tarshish, O., Canny, J.: Livenotes: A System for Cooperative and Augmented Note-Taking in Lectures. In: Proceedings of CHI 2005. (2005) 531-540

3. Ratto, M., Shapiro, R.B., Truong, T.M., Griswold, W.G.: The ActiveClass Project: Experiments in Encouraging Classroom Participation. In: Proceedings of CSCL 2003. (2003) 477-486

4. Yang, J.C., Chen, C.H.: Design of Inquiry Learning Activity Using Wireless and Mobile Technologies. In: Proceedings of ICALT 2006. (2006) 398-402

5. Yatani, K., Sugimoto, M., Kusunoki, F.: Musex: A System for Supporting Children's Collaborative Learning in a Museum with PDAs. In: Proceedings of WMTE 2004. (2004) 109-113

6. Oviatt, S., Arthur, A., Cohen, J.: Quiet Interfaces that Help Students Think. In: Proceedings of UIST 2006. (2006) 191-200

7. Miura, M., Kunifuji, S., Shizuki, B., Tanaka, J.: Augmented Classroom: A PaperCentric Approach for Collaborative Learning System. In: Proceedings of 2nd Int. Symposium on Ubiquitous Computing Systems (UCS2004), LNCS 3598. (2004) $104-116$

8. Miura, M., Kunifuji, S., Sakamoto, Y.: AirTransNote: An Instant Note Sharing and Reproducing System to Support Students Learning. In: Proceedings of ICALT 2007. (2007) (to appear)

9. Cooperstock, J.R.: The Classroom of the Future: Enhancing Education through Augmented Reality. In: Proceedings of HCI International 2001. (2001) 688-692

10. Shi, Y., Xie, W., Xu, G.: Smart Remote Classroom: Creating a Revolutionary Real-Time Interactive Distance Learning System. In: Proceedings of ICWL 2002, LNCS 2436. (2002) 130-141

11. Vila, X., Riera, A., Sánchez, E., Lama, M., Moreno, D.: A PDA-based Interface for a Computer Supported Educational System. In: Proceedings of ICALT 2003. (2003) $12-16$

12. Liu, C.C., Kao, L.C.: Handheld Devices with Large Shared Display Groupware: Tools to Facilitate Group Communication in One-to-One Collaborative Learning Activities. In: Proceedings of WMTE 2005. (2005) 128-135 